\title{
Del dicho al hecho en una propuesta sobre ecosistemas contextualizada en el huerto escolar
}

\author{
Ma José Sáez Bondía ${ }^{1}$, Pedro Lucha López ${ }^{1}$, Ana María Claver Giménez ${ }^{2}$, Ángel \\ ${\text { Arasanz } \text { Broto }^{2} \text { y Rafael Iráizoz Esteban }}^{2 \text { y } 3}$ \\ ${ }^{1}$ Dpto. Didáctica de las Ciencias Experimentales. Universidad de Zaragoza. ${ }^{2} \mathrm{CEIP}$ EI \\ Parque. ${ }^{3}$ Dpto. Ciencias de la Educación. Universidad de Zaragoza.
}

[Recibido el 5 de octubre de 2017, aceptado el 11 de diciembre de 2017]

El presente trabajo recoge el desarrollo de una propuesta sobre ecosistemas desde su diseño y planificación hasta su aplicación. Dicha propuesta, contextualizada en el huerto escolar, se fundamenta en el desarrollo del pensamiento sistémico de alumnos de 60 de Primaria y surge de la colaboración entre profesores universitarios y una maestra de Primaria. La implementación de secuencias que implican cierto grado de investigación por parte de los alumnos hace que durante el desarrollo de la actividad surjan dilemas por parte de los maestros sobre cómo continuar con lo planificado. Las decisiones tomadas para resolverlos determinan la secuencia llevada a cabo y constituyen nuevas oportunidades de aprendizaje para los estudiantes de primaria, al mismo tiempo que permiten a los maestros y colaboradores reflexionar sobre las decisiones tomadas de cara a futuras programaciones. Finalmente, se discuten los efectos del desarrollo de esta propuesta en la enseñanza y aprendizaje de los ecosistemas y en el pensamiento sistémico de los alumnos.

Palabras clave. Ecosistemas; pensamiento sistémico; huerto escolar; educación primaria

\section{From words to action: an activity on ecosystems framed in the school garden}

This paper narrates the development of a learning sequence about ecosystems, from its planning till it was carried out. The sequence uses the school garden as a resource, it's focused on the development of system thinking among $6^{\text {th }}$ grade students and it is the result of collaboration between researchers from the University and school teachers. Teaching sequences including some extent of students' research lead to dilemmas among teachers about how to continue with the programmed plan. Decisions taken to solve them condition the final sequence carried out, constitute new learning opportunities for students, allow teachers to reflect on the decisions taken and should have implications on teachers' programming. Finally, the effects of the implemented sequence on the students' system-thinking and on the acquisition of the ideas about ecosystems are discussed.

Keywords. Ecosystems, system thinking, school garden, primary education.

Para citar el artículo. Sáez Bondía, M.J., Lucha López, P., Claver Giménez, A.M., Arasanz Broto, A. y Iráizoz Esteban, R. (2017). Del dicho al hecho en una propuesta sobre ecosistemas contextualizada en el huerto escolar. Ápice. Revista de Educación Científica, 1(2), 47-57. DOI: https://doi.org/10.17979/arec.2017.1.2.3085

Contacto.msaezbo@unizar.es, plucha@unizar.es 


\section{Introducción}

Son numerosos los materiales y orientaciones didácticas publicados en torno al huerto escolar que hacen que el uso de este recurso llegue a los diferentes niveles educativos (Eugenio y Aragón, 2016). Sin embargo, esa variedad de propuestas concretas, en muchas ocasiones, tratan sobre experiencias puntuales en el huerto que requieren de cierta contextualización cuando tienen que ser trasladadas al aula (Desmond, Grieshop y Subramaniam, 2002). De este modo, son diversas las temáticas desde las que se trabaja con el huerto escolar en diferentes propuestas, como por ejemplo la alimentación, la agricultura, la ecología o sus implicaciones medioambientales. Independientemente de la temática a trabajar, las propuestas en torno al huerto escolar promueven frecuentemente la manipulación y la realización de pequeñas investigaciones (véase Perelló, 2010; Bergada, 2013, Eugenio y Aragón, 2016).

Ahora bien, si tomamos como referencia estas propuestas con el objetivo de ser aplicadas en un aula concreta, nos encontramos con determinados factores que hacen que el rumbo de las mismas siga caminos inesperados (Baena, 2000). La pretensión de este trabajo no es estudiar cuáles son esos factores, sino relatar el desarrollo de una propuesta contextualizada en el huerto escolar como ecosistema, desde su planteamiento hasta su realización. Para ello, mostramos el contexto en el que se enmarcó dicha propuesta, los acuerdos a los que se llegaron en el diseño y planificación de la misma ("lo dicho"), lo que sucedió durante la aplicación de la misma ("lo hecho"), para, finalmente, discutir la distancia existente entre "lo dicho" y "lo hecho" y sus implicaciones.

\section{Contexto y participantes}

El presente trabajo surge de una colaboración entre profesores de Didáctica de las Ciencias Experimentales (en adelante, PDC) y dos maestros y una maestra del CEIP El Parque de la ciudad de Huesca de dos vías que impartían la asignatura de Ciencias Naturales en 50 y 6ㅇ de Primaria. A partir de una reunión inicial se decidió diseñar una misma propuesta para los dos cursos. Así, los tres maestros acordaron que una de las temáticas con las que tenían más dificultades y en la que por lo tanto preferían centrar la colaboración eran "Los Ecosistemas". En este trabajo nos centramos en el diseño acordado y la aplicación llevada a cabo por la maestra de una de las aulas de sexto. Hemos decidido limitarnos a relatar la implementación llevada a cabo por una de las maestras porque permite abordar con un mayor detalle y profundidad tanto la praxis como la discusión.

El modo en el que esta maestra, especialista en francés y tutora del aula, se enfrentaba habitualmente a sus clases de ciencias se basaba casi exclusivamente en el uso del libro de texto, ya que sentía cierta inseguridad hacia la asignatura. No obstante, la forma en la que gestionaba el aula permitía que los alumnos participasen de forma activa en sus clases. En este sentido, los alumnos, de un modo organizado, planteaban cuestiones sin miedo y proponían respuestas a las mismas debatiendo, bien con todo el grupo-clase $o$ en pequeños grupos, que es como se disponían en las mesas.

\section{Lo dicho: el planteamiento de una propuesta sobre el huerto escolar como ecosistema para fomentar el pensamiento sistémico}

¿Cómo abordar el estudio de los ecosistemas?

No cabe duda de que los fenómenos naturales son, en su mayoría, complejos. Se trata 
de sistemas dinámicos que cambian, se auto-organizan y se adaptan continuamente (Jacobson y Wilensky, 2006). Es más, por sí solos los seres vivos son sistemas complicados que, a su vez, forman parte de sistemas de poblaciones, comunidades, ecosistemas y finalmente, de toda la biosfera (Riess y Mischo, 2010). Tal vez sea este el motivo por el cual resulta difícil introducir el concepto de ecosistema en las aulas, ya que no se trata de un concepto exclusivamente descriptivo, no admite simplificaciones y necesita la consideración no solo de los aspectos espaciales sino también de los temporales (Del Carmen, 1999).

Por tanto, la comprensión del funcionamiento de los ecosistemas requiere que los alumnos hayan adquirido la capacidad de pensamiento sistémico (Ben-Zvi Assaraf y Orion, 2005). Para ello, estos autores, sin distanciarse mucho de las recomendaciones propuestas por Del Carmen (1999) sobre la introducción progresiva de la enseñanza de los ecosistemas a través de cuestiones, plantean que el proceso de enseñanza consista en una secuencia de fases que sigan un orden jerárquico. Dicha jerarquía se compone de tres niveles, cada uno de los cuales incluye una serie de características, también jerárquicas, que, una vez adquiridas, conducen a un pensamiento sistémico (véase relación entre ambos enfoques en tabla 1).

Tabla 1. Relación entre el modelo jerárquico del pensamiento sistémico (System Thinking Hierarchical (STH) Model de Ben-Zvi Assaraf y Orion, 2005) y las recomendaciones de Del Carmen (1999)

\begin{tabular}{|c|c|c|}
\hline $\begin{array}{l}\text { Niveles del } \\
\text { modelo STH }\end{array}$ & $\begin{array}{c}\text { Características jerárquicas en cada } \\
\text { nivel STH }\end{array}$ & $\begin{array}{c}\text { Recomendaciones de introducción } \\
\text { progresiva (Del Carmen, 1999) }\end{array}$ \\
\hline $\begin{array}{l}\text { Nivel 1: } \\
\text { Análisis de los } \\
\text { componentes } \\
\text { del sistema }\end{array}$ & $\begin{array}{l}\text { 1.Identificar los elementos de un } \\
\text { sistema y sus procesos }\end{array}$ & $\begin{array}{l}\text { ¿Cómo es el ecosistema? } \\
\text { (Límites, caracterización de factores } \\
\text { abióticos y bióticos, estudio de la } \\
\text { diversidad y abundancia de especies, } \\
\text { ocupación del espacio por los } \\
\text { organismos) }\end{array}$ \\
\hline $\begin{array}{l}\text { Nivel 2: } \\
\text { Síntesis de los } \\
\text { componentes } \\
\text { del sistema }\end{array}$ & $\begin{array}{l}\text { 2.Identificar relaciones simples } \\
\text { entre dichos elementos } \\
\text { 3.Identificar relaciones dinámicas } \\
\text { dentro del sistema } \\
\text { 4.Organizar los elementos y } \\
\text { procesos del sistema y sus } \\
\text { interacciones } \\
\text { 5.Identificar ciclos de materia y } \\
\text { energía en el sistema }\end{array}$ & $\begin{array}{l}\text { ¿Cómo es el ecosistema? } \\
\text { Relaciones entre organismos del } \\
\text { ecosistema, adaptaciones. }\end{array}$ \\
\hline $\begin{array}{l}\text { Nivel 3: } \\
\text { Aplicación } \\
\text { (transferencia) }\end{array}$ & $\begin{array}{l}\text { 6.Reconocer los elementos } \\
\text { escondidos del sistema } \\
\text { 7.Hacer generalizaciones } \\
\text { 8.Pensar temporalmente }\end{array}$ & $\begin{array}{l}\text { ¿Cómo cambia el ecosistema con el } \\
\text { tiempo? (ritmos biológicos: diurno, } \\
\text { estacional y sucesiones) } \\
\text { ¿Cómo interactúa con otros } \\
\text { ecosistemas? }\end{array}$ \\
\hline
\end{tabular}

En cuanto al tipo de ecosistema que estudiar, Del Carmen (1999) propone comenzar el estudio de ecosistemas del entorno más próximo, ya que son los que los alumnos conocen mejor. Así, en el caso que nos ocupa, aprovechando que el centro disponía de un pequeño huerto escolar, decidimos contextualizar la propuesta en el ecosistema del huerto escolar. 


\section{Diseño y planificación de la actividad}

En la tabla 2 se muestra el diseño y planificación de la propuesta que se acordó con la maestra de 6 o de Primaria siguiendo las recomendaciones de introducción progresiva expuestas anteriormente.

Tabla 2. Diseño y planificación de la actividad: "El ecosistema del huerto del cole" y su relación con el modelo STH (Ben-Zvi Assaraf y Orion, 2005)

\begin{tabular}{|c|c|c|}
\hline $\begin{array}{c}\text { Nivel } \\
\text { STH }\end{array}$ & Cuestiones guía & Número de sesión, duración acordada y descripción \\
\hline 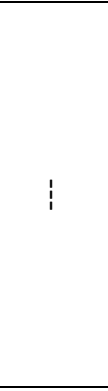 & $\begin{array}{l}\text { ¿Qué conocéis del huerto del } \\
\text { cole? ¿Qué seres vivos } \\
\text { podemos encontrar? ¿Cómo } \\
\text { podemos comprobarlo? }\end{array}$ & $\begin{array}{l}\text { Sesión } 1 \text { (45 min) } \\
\text { Recordamos, a partir de visitas anteriores al huerto, } \\
\text { qué nos podemos encontrar. } \\
\text { Hacemos una lista y diferenciamos los seres vivos de } \\
\text { los elementos inertes. Realizamos sub-clasificaciones } \\
\text { dentro de los seres vivos (animales y vegetales). } \\
\text { Planteamos un modo de salir a comprobar nuestras } \\
\text { predicciones sobre qué hay en el huerto: materiales } \\
\text { que necesitamos y cuándo salimos }\end{array}$ \\
\hline 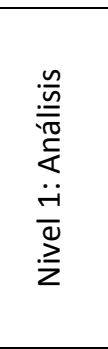 & $\begin{array}{l}\text { ¿Cómo son los seres vivos del } \\
\text { huerto? ¿Cómo se llaman? } \\
\text { ¿De qué se alimentan? }\end{array}$ & $\begin{array}{l}\text { Sesión } 2 \text { ( } 45 \text { minutos) } \\
\text { Salimos al huerto y anotamos lo que hemos visto, } \\
\text { tomamos muestras de algunos seres vivos. } \\
\text { Sesión } 3 \text { ( } 2 \text { horas y } 30 \text { minutos) } \\
\text { En el laboratorio con ayuda de la lupa binocular, los } \\
\text { describimos. Posteriormente los identificamos. } \\
\text { Conocemos acerca de esos seres vivos buscando } \\
\text { información (sus necesidades, su hábitat,...) }\end{array}$ \\
\hline 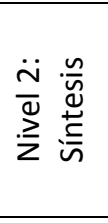 & $\begin{array}{l}\text { ¿Cómo se relacionan entre sí } \\
\text { los seres vivos del huerto? } \\
\text { ¿Qué beneficios obtienen } \\
\text { unos de otros? ¿En qué se } \\
\text { perjudican? }\end{array}$ & $\begin{array}{l}\text { Sesión } 4 \text { ( } 45 \text { minutos) } \\
\text { Relacionamos los seres vivos del huerto a través de } \\
\text { una red trófica y hablamos de las relaciones entre } \\
\text { ellos. }\end{array}$ \\
\hline 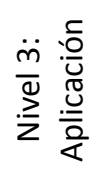 & $\begin{array}{l}\text { ¿Qué pasaría si algunos de } \\
\text { estos organismos no } \\
\text { estuviese en el huerto? }\end{array}$ & $\begin{array}{l}\text { Sesión } 5 \text { ( } 45 \text { minutos) } \\
\text { Argumentamos sobre lo que pasaría si algunos de los } \\
\text { seres vivos del huerto no estuviesen. }\end{array}$ \\
\hline
\end{tabular}

Dicha propuesta estaba planteada para ser aplicada en 5 sesiones de duración variable, sumando unas 6 horas, en la asignatura de Ciencias Naturales. No obstante, la maestra se mostró flexible a la hora de emplear horas de otras de las asignaturas que impartía.

Considerando la programación del curso, se acordó comenzar la aplicación de la propuesta en el mes de febrero. El invierno no es una buena estación para realizar el trabajo de campo de un ecosistema porque muchos seres vivos están en estado latente. Sin embargo, ante la imposibilidad de retrasar la implementación, se contemplaron soluciones a los problemas que, inicialmente, se previó que podían surgir.

El huerto escolar del CEIP El Parque se encuentra en una zona sombría, separada del patio del recreo por una valla metálica. Consta de tres pequeños maceteros de unos dos metros cuadrados cada uno. Durante el otoño anterior a la puesta en marcha de la propuesta se habían plantado en el huerto: trigo, coles (repollo), borraja, acelgas y lechugas. En febrero era probable que no encontrásemos ni mucha diversidad, ni mucha abundancia de seres vivos observables a simple vista, así que se propuso como medida 
preventiva introducir lombrices en los maceteros antes de la salida, para que los alumnos las encontraran.

\section{Del dicho al hecho: el desarrollo de la propuesta}

La comparación de la tabla 2 con el anexo 1, permite comprobar cuáles de las actividades diseñadas fueron implementadas según lo planificado y qué sesiones se añadieron a la secuencia diseñada a raíz de los dilemas y de las decisiones tomadas durante la fase de desarrollo (sesiones adicionales 1 y 2). Mostramos a continuación los tres dilemas que mayores repercusiones tuvieron en la actividad, así como la importancia de la colaboración entre la maestra y los PDC en la toma de dichas decisiones.

\section{Tres momentos clave en el desarrollo}

Finalmente la propuesta contó con siete sesiones y unas ocho horas de duración. Mostramos a continuación esos momentos clave.

\section{La preocupación por mantener vivos a los seres vivos muestreados}

Durante la salida, los alumnos encontraron y muestrearon una pequeña larva de tijereta, varias escolopendras, lombrices y una babosa. Las especies vegetales no se muestrearon sino que fueron identificadas in situ (figura 1).

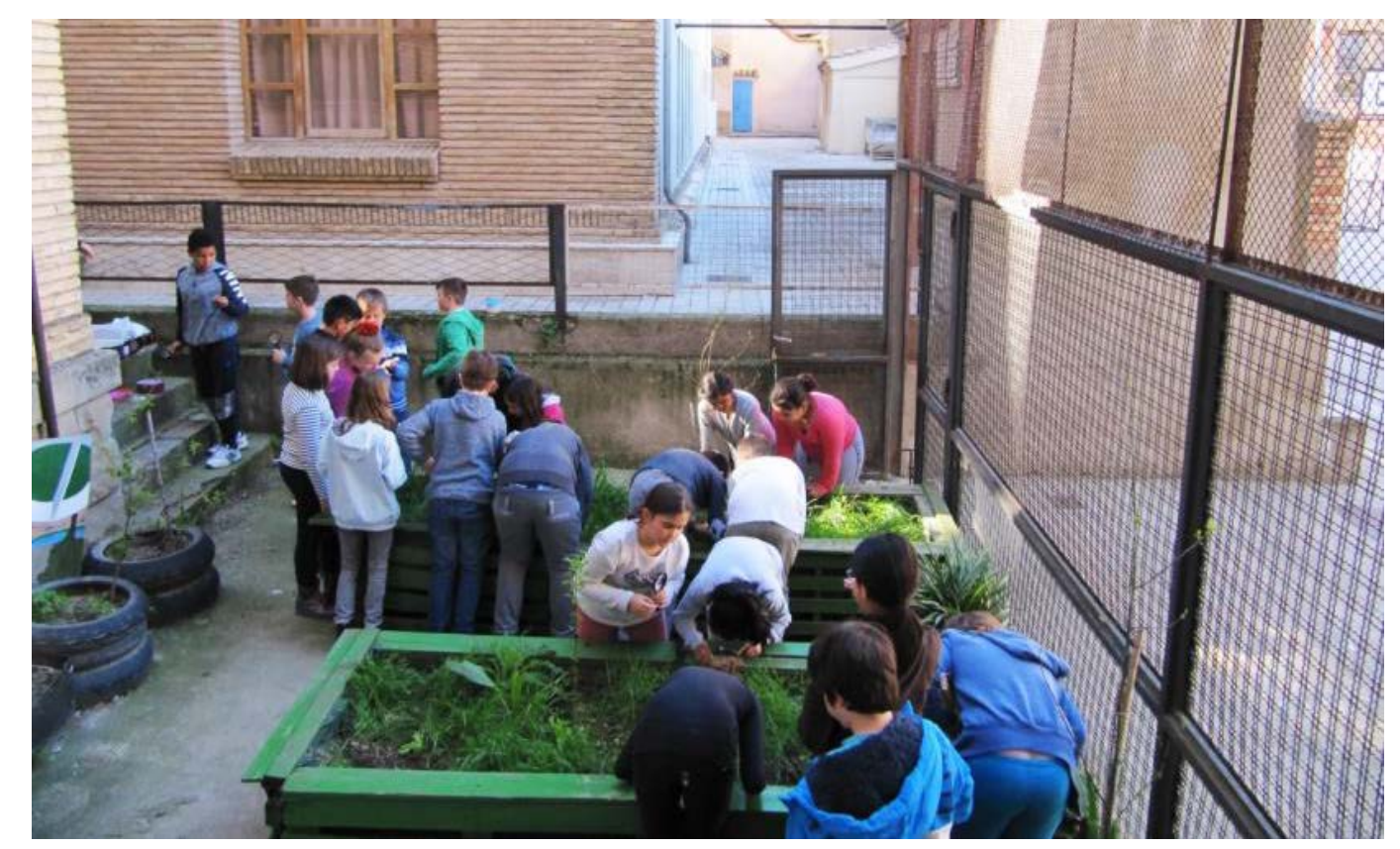

Figura 1. Alumnos en el huerto escolar durante la sesión 2

Los alumnos regresaron al aula con sus pequeños animales y mostraron su preocupación por que muriesen. Inicialmente, los PDC, a petición de los alumnos, les dieron pistas sobre cómo podían vivir en clase, no obstante, algunos de los animales esa misma tarde habían muerto. La maestra y los alumnos, mandaron a uno de los PDC un correo muy preocupados y éste contestó en cuanto pudo (figura 2). 


\begin{tabular}{|c|c|}
\hline & $\begin{array}{l}\text { Respuesta dada por uno de los PDC } \\
\text { Buenos díos clase de } 6^{\circ} \mathrm{B}\end{array}$ \\
\hline \multirow{7}{*}{$\begin{array}{l}\text { Correo enviado por los alumnos a uno de los PDC } \\
\text { Buenos días Pedro, } \\
\text { Ya hace dos días que tenemos en clase a los animales que } \\
\text { recogimos del huerto. } \\
\text { El ciempiés había muerto aquella misma tarde cuando } \\
\text { entramos a las } 15 \text { horas. Empieza a desmembrarse. } \\
\text { La lombriz ha aparecido esta mañana bajo la mesa, casi } \\
\text { seca. La hemos humedecido y parece hacer revivido. } \\
\text { La babosa continúa viva, no sabemos por cuanto tiempo. } \\
\text { Nos preguntamos si a vosotros os vendría bien adelantar la } \\
\text { sesión de laborario para poder dejar en libertad a los } \\
\text { animales que queden vivos. Nos están dando mucha pena. } \\
\text { Un saludo. } \\
\text { La clase de } 6^{\circ} \text { B } \\
\text { P.D. Estaremos dispuestos cualquier mañana. }\end{array}$} & $\begin{array}{l}\text { Es una pena lo del ciempiés, pero no os preocupéis porque cada año, un } \\
\text { ciempiés como el que se ha muerto, pone entre } 20 \text { y } 30 \text { huevos de los que } \\
\text { saldrán otros tantos pequeños ciempiés. Eso hace entre } 100 \text { y } 150 \text { nuevos } \\
\text { ciempiés, por cada ciempiés adulto, cada } 5 \text { años. }\end{array}$ \\
\hline & $\begin{array}{l}\text { Olvidé deciros que a las lombrices no les gusta la luz y para que estén a } \\
\text { gusto es recomendable tapar el recipiente donde viven con una tela } \\
\text { cualquier otra cosa que impida la entrada de luz. De esta manera, estará } \\
\text { contenta en la tierra y no se escapará en busca de lugares oscuros iEs } \\
\text { muy importante también que la tierra esté siempre húmedal }\end{array}$ \\
\hline & $\begin{array}{l}\text { Si la babosa tiene lechuga y no hace demasiado frío esperemos que } \\
\text { sobreviva. }\end{array}$ \\
\hline & $\begin{array}{l}\text { Para que la hoja de lechuga que come no se seque, la podemos poner en } \\
\text { un pequeño jarrón con agua. Es importante que el jarrón sea pequeño y } \\
\text { que no haya mucha agua porque si no, la babosa puede caer al agua y } \\
\text { ahogarse. }\end{array}$ \\
\hline & $\begin{array}{l}\text { La semana que viene yo podría acompañaros al laboratorio cualquier } \\
\text { mañana. De hecho, está todo preparado desde que subió la clase de } 6^{\circ} \text { A } \\
\text { Se que a María José le hace mucha ilusión acompañarnos en el } \\
\text { laboratorio el lunes o el martes por la mañana. La incluyo entre los } \\
\text { destinatarios de este mensaje para que nos de su opinión. }\end{array}$ \\
\hline & Un fuerte abrazo y hasta pronto \\
\hline & Pedro \\
\hline
\end{tabular}

Figura 2. Correos electrónicos para solucionar el problema: ¡Nuestros animales se mueren!

Tras el intercambio de correos y ante la demanda de una de las alumnas de "llevar su casa a clase", los alumnos, con ayuda de uno de los PDC, construyeron un terrario en clase. De este modo, no solo mantendrían vivos a "sus animales" sino que podrían observarlos todos los días.

Como consecuencia de esta decisión, durante el desarrollo de la propuesta, los alumnos pudieron ver cómo las lombrices removían la tierra y cómo se alimentaba la babosa que además, puso huevos. Observaron los huevos en la lupa, vieron nacer a las babosas y las contaban frecuentemente para comprobar si eclosionaban nuevos huevos o si moría alguna. Estas observaciones hicieron que se planteasen cuestiones sobre la reproducción de las babosas y las lombrices.

\section{Dificultades para identificar: ¿Cómo se llama el "bicho" de los 23 pares de patas?}

Puesto que algunos de los animales muestreados habían muerto, se decidió coger nuevas lombrices y escolopendras para observar en la sesión 3 de laboratorio. Durante esta sesión, los alumnos se familiarizaron con el uso de la lupa binocular, observaron lombrices vivas a través de ésta y contaron las patas de la escolopendra. Sin embargo, a la hora de identificar los animales desconocidos (la larva de tijereta y la escolopendra), hubo problemas: los libros de identificación de fauna de los que disponíamos eran muy complejos para alumnos de Primaria y además, no estaban familiarizados con su uso. Ante este problema, la sesión se basó en la observación y descripción de los ejemplares.

Tras la sesión, hablamos con la maestra sobre las dificultades de los alumnos para identificar los animales y se propuso que los alumnos nos mandasen otro correo con una descripción de los seres vivos desconocidos para que nosotros les dijéramos sus nombres así como los motivos por los que habíamos averiguado que se trataba de esos animales en concreto. De esta manera podríamos continuar con la elaboración de la red trófica en la siguiente sesión (figura 3). 


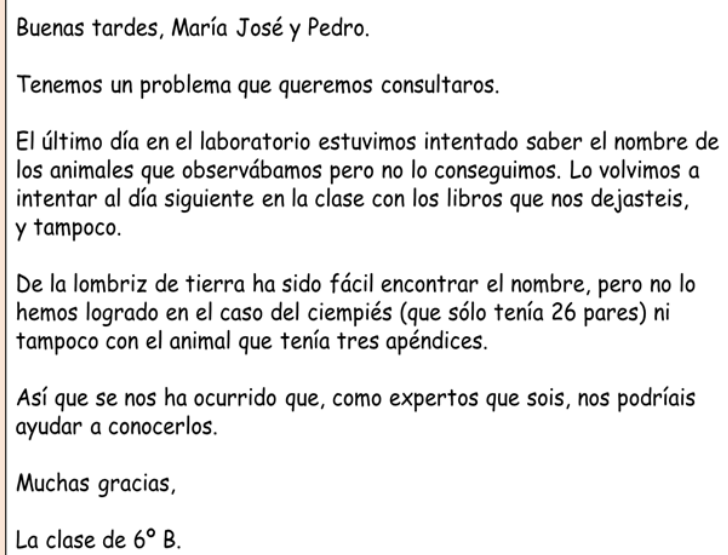

Figura 3. Correo electrónico recibido ¿cómo se llaman algunos de los animales del huerto?

La descripción dada no fue lo suficientemente exhaustiva como para motivar una identificación justificada, así que decidimos adaptar una clave dicotómica sencilla para que los alumnos en una sesión adicional identificasen "sus animales" (figura 4). En dicha sesión, se habló sobre el origen de los nombres científicos y se aprendió a manejar una clave sencilla para identificar la larva de tijereta (otros insectos) y la escolopendra.

Clave dicotómica de identificación de algunos invertebrados presentes en el jardín

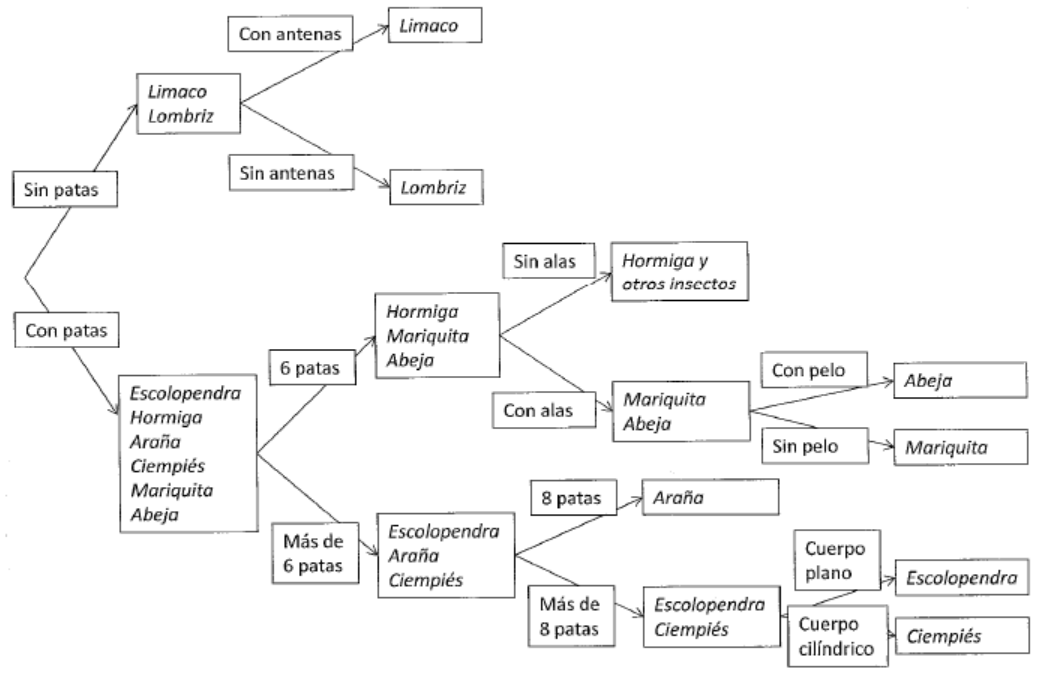

Figura 4. Clave de identificación empleada

\section{Dudas en la red trófica y cómo hacer predicciones sobre ella.}

Las dificultades para identificar los ejemplares y la presencia del terrario en clase, hicieron que el objetivo de la actividad: investigar el huerto como un ecosistema en el que existen relaciones entre los componentes del mismo, se desdibujara. Así, decidimos volver de nuevo al huerto para recordar dicho objetivo y para retomar, una vez conocidos los nombres de los seres vivos y su alimentación, la elaboración de la red trófica del ecosistema del huerto. En ese curso los alumnos no habían recibido instrucción alguna sobre qué es una red trófica y no se les demandó que hicieran una 
del ecosistema del huerto, sino que lo que se les pidió es que tratasen de relacionar los diferentes seres vivos hasta llegar, bajo acuerdos, a la elaboración de la misma (figura 5). Durante la sesión, surgía el dilema de "cómo y dónde" poner a la lombriz, o en qué sentido poner las flechas. A través de la puesta en común en clase se acordó poner en el cajón "tierra" a la lombriz y que ahí iban los restos de los seres vivos cuando morían. En cuanto al sentido de las flechas, surgió el tema del flujo de energía como un modo de representación.

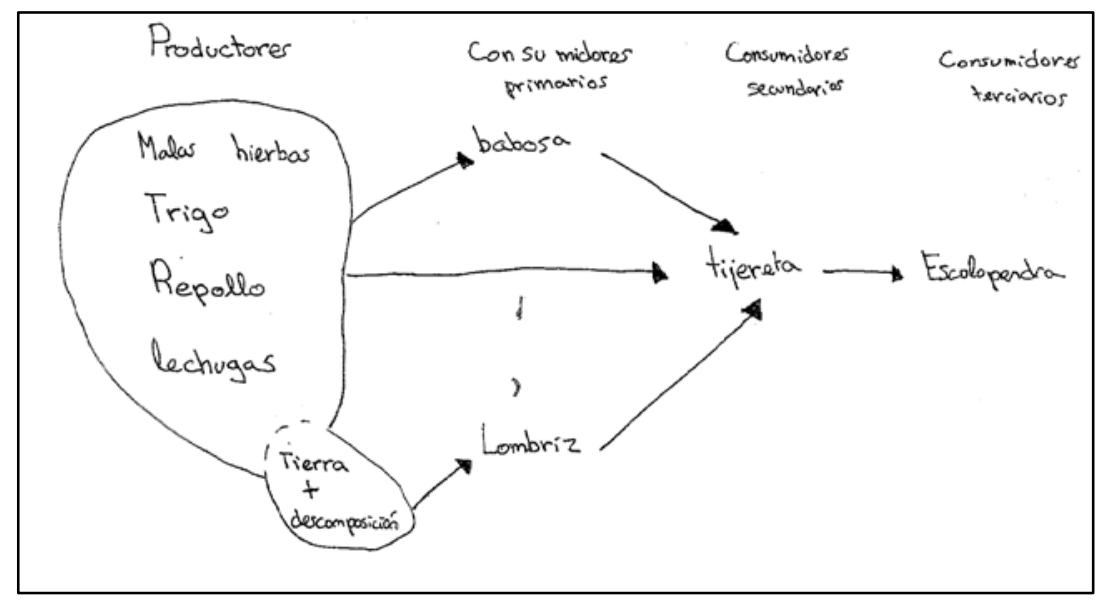

Figura 5. La red trófica del huerto del colegio acordada

Para finalizar, en la siguiente sesión se planteó la realización de predicciones acerca de qué pasaría si uno de los seres vivos no estuviese. Para limitar el número de elementos a incorporar en las argumentaciones, decidimos focalizar las predicciones con cuestiones del tipo: ¿Qué le pasaría a la babosa si no hubiese lechuga? ¿Y si no hubiese tijeretas?

La mayoría de los argumentos dados por los alumnos acababan con versiones "catastrofistas", considerando todos los elementos de su "sistema", considerando no solo las relaciones de depredación, sino también de competencia. Incluso, algunos alumnos consideraban que el huerto de por sí se relacionaba con otros ecosistemas como el parque próximo al colegio. Mostramos una de las respuestas escritas dada por un grupo de alumnos en la figura 6 .

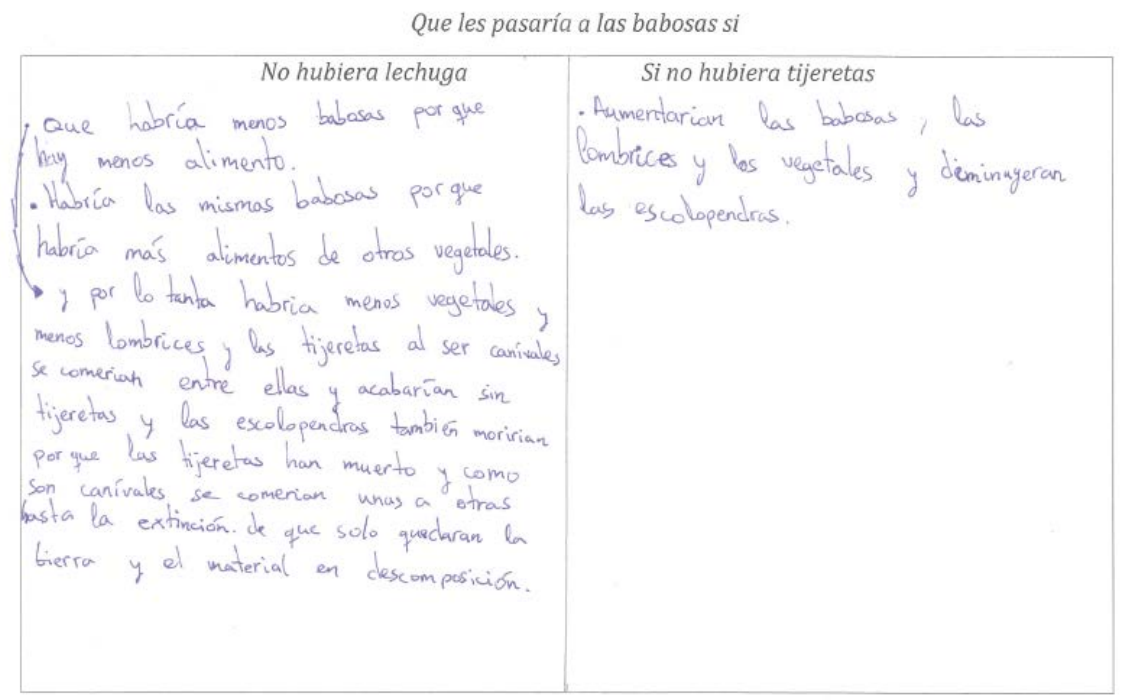

Figura 6. Respuestas de los alumnos sobre qué pasaría si... 


\section{La importancia de una coordinación entre la maestra y los PDC}

Las actividades desarrolladas fueron dirigidas por la maestra, no obstante, para registrar lo qué sucedía durante el desarrollo de las mismas, los dos PDC asistieron a las sesiones en el papel de "oyentes" y colaborando en algunas ocasiones. De este modo, redactaban, sesión a sesión, un diario descriptivo. Dicho diario se utilizó para identificar los problemas que surgieron y decidir, junto con la maestra, las posibles soluciones a los mismos.

Asimismo, a partir del dilema surgido en la sesión 2 (¿Cómo mantenemos vivos a los seres vivos?), la maestra decidió acudir a los PDC para solicitar ayuda "técnica". La actuación en calidad de "expertos en ecosistemas" de los dos PDC para atender a esa demanda los consolidó en ese rol y a partir de ese momento compaginaron ambos roles: el de "oyentes" y el de "expertos" (como por ejemplo, de nuevo en la sesión 3: ¿Cómo se llama este bicho?).

Así, la coordinación entre la maestra y los PDC constituyó un pilar fundamental en la toma de decisiones a lo largo de la propuesta planteada y el modo en el que finalmente se desarrolló.

\section{Implicaciones para la enseñanza y aprendizaje de las ciencias}

El relato de la secuencia de actividades sobre ecosistemas constata que existen diferencias entre lo programado y lo implementado cuando se trata de llevar a cabo actividades donde los alumnos realizan pequeñas investigaciones.

La conversación reflexiva entre los PDC y la maestra en la toma de decisiones frente a situaciones de duda durante el desarrollo de la actividad presentada ha favorecido la creación de nuevas oportunidades de aprendizaje. Por ejemplo, montar el terrario, bajo la demanda de los alumnos, favoreció que estos conociesen algo más sobre las lombrices y las babosas e hiciesen observaciones periódicas utilizando la lupa binocular.

Además, pensar sobre las decisiones tomadas nos permite plantear mejoras en propuestas similares a la presentada, considerando, por ejemplo, la necesidad de dar tiempo a los estudiantes para que adquieran habilidades de identificación de ejemplares o aprendan a diferenciar seres vivos de lo que no lo son.

También se ha constatado la dificultad intrínseca que entraña la comprensión de los ecosistemas y las destrezas cognitivas que requiere por parte de los alumnos. A pesar de ello, los alumnos, con los seres vivos caracterizados en el huerto, fueron capaces de construir una red trófica en la que no solo se consideraron las relaciones de depredación, sino también las de competencia, habiendo una relación que iba más allá de una concepción lineal de las interacciones entre los elementos del ecosistema (Gil Quílez y Martínez Peña, 1992).

De este modo, el grado de comprensión del ecosistema investigado por parte de algunos alumnos sugiere que estos están en posesión de habilidades cognitivas situadas en la parte alta de la pirámide que conduce al pensamiento sistémico, por ejemplo siendo capaces de pensar temporalmente tras haber superado la identificación de los elementos del sistema y algunas de sus relaciones (Ben-Zvi Assaraff y Orion, 2005).

\section{Agradecimientos}

Grupo Consolidado de Investigación Aplicada BEAGLE (Gobierno de Aragón y Fondo Social Europeo); miembro del Instituto de Investigación en Ciencias Ambientales de 
Aragón (IU-CA/UNIZAR); Proyectos EDU2016-76743-P (MEC) y PIIDUZ_16_108 (UNIZAR); CEIP EI Parque.

\section{Referencias}

Baena, M. (2000). Pensamiento y acción en la enseñanza de las ciencias. Enseñanza de Las Ciencias, 18(2), 217-226.

Ben-Zvi Assaraf, O. y Orion, N. (2005). Development of system thinking skills in the context of earth system education. Journal of Research in Science Teaching, 42(5), 518-560.

Bergada, R. (2013). Un ecosistema: el huerto escolar. Padres y maestros, 349, 41-46.

Del Carmen, L. (1999). El estudio de los ecosistemas. Alambique. Didáctica de las Ciencias Experimentales, 20, 47-54.

Desmond D., Grieshop J. y Subramaniam A. (2002) Revisiting garden-based learning in basic education. Paris: International Institute for Educational Planning (IIEP). Recuperado de: http://www.fao.org/3/a-aj462e.pdf

Eugenio M. y Aragón, L. (2016) Experiencias en torno al huerto ecológico como recurso didáctico y contexto de aprendizaje en la formación inicial de maestros/as de Infantil. Revista Eureka sobre Enseñanza y Divulgación de las Ciencias, 13 (3), 667679. Recuperado de: http://hdl.handle.net/10498/18504

Gil Quílez, M.J. y Martínez Peña, B. (1992). Problemática de la enseñanza/aprendizaje de la ecología. Revista interuniversitaria de formación del profesorado, 14, 67-70.

Jacobson, M.J. y Wilensky, U. (2006). Complex systems in education: Scientific and educational importance and implications for the learning sciences. The Journal of the learning sciences, 15(1), 45-52.

Perelló Mulet, J. (2010). L'hort escolar: Un programa científic d'aprenentatge. Recursos i Recerca Educativa de les Illes Balears, 1. 155-176. Recuperado de http://www.innovib.cat/numero-1/pdfs/9-pre-op.pdf

Riess, W. y Mischo, C. (2010). Promoting Systems Thinking through Biology Lessons. International Journal of Science Education, 32, 705-725. 
Anexo 1. Secuencia de dilemas y decisiones tomadas y que dieron lugar a "lo hecho", lo que realmente sucedió. El cuadro superior de cada par contiene los dilemas y el cuadro inferior las decisiones que se tomaron. Para cada pareja se indica, al lado, el momento en el que se dio durante la secuencia. Los cuadrantes con fondo coloreado corresponden con las sesiones inicialmente planificadas.

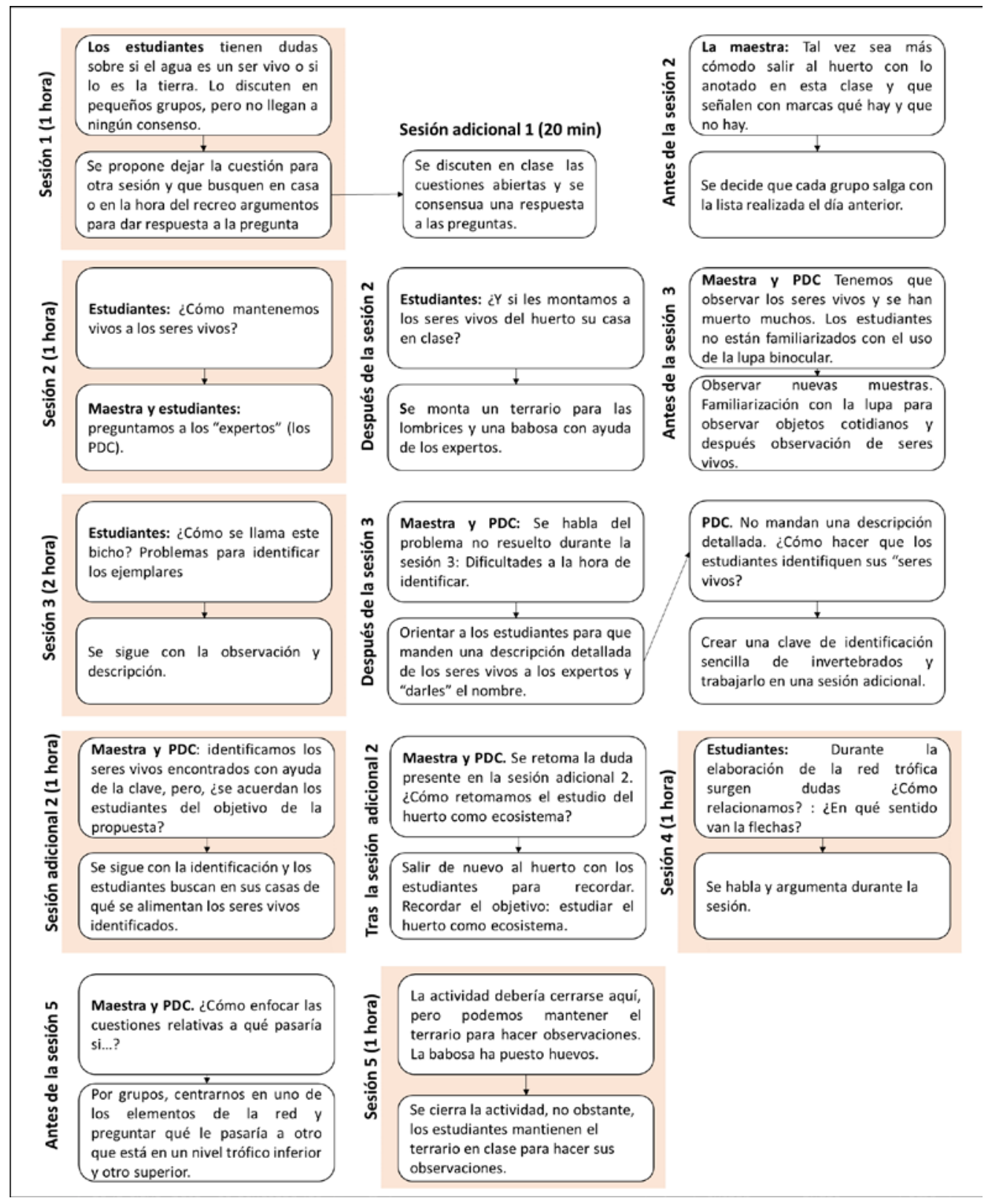

